\title{
A Parametric Modeling Instructional Tutorial for Teaching Visualization of Engineering Mechanics
}

\author{
Pam Schumacher, $\mathrm{MS}^{1}$, and Winston Sealy, $\mathrm{PhD}^{2}$ \\ ${ }^{1}$ Saint Paul College, St. Paul, USA, Pam.schumacher@saintpaul.edu \\ 2 Minnesota State University, Mankato, USA, Winston.sealy@mnsu.edu
}

\begin{abstract}
Communication is paramount for relaying information. Manufacturers rely heavily on business practices that are clearly communicated. As new products are designed, drawings and sketches are utilized to communicate form, fit, and function. Visualization techniques and practices are critical for understanding and effective communication. This paper presents an instructional tutorial for utilizing parametric modeling to enhance the design and visualization of engineering mechanics. Real world examples are modeled and simulated using Creo Parametric ver. 4.0.
\end{abstract}

Keywords_-Visualization, Parametric Modeling, Engineering Mechanics, Instructional Tutorial.

\section{INTRODUCTION}

Engineers and designers communicate through drawings and sketches, however, without proper visualization, effective communication can be strained [1]. Visualization is important for engineering students to be able to solve 2D and 3D problems. It is important to visualize how objects look, how they move and how they deform, especially when forces and moments are applied.

US Air Force Academy of Mechanical Engineering published a study in 2001 highlighting the failure of multimedia in the classroom [2]. The study delineated realworld examples of several engineering mechanics concepts of varying levels of difficulty. As such, the study focused primarily on the application of torsion, bending and combined loads. The examples were presented using PowerPoint, and computer simulations of finite element plots depicting stress distributions. The hypothesis was that these real-world examples and multimedia presentations would increase the interest and satisfaction of the lecture for the students. The opposite was actually found to be true. A five-question survey about the lecture indicated a sharp decrease in satisfaction for the lectures containing the real world examples and simulations as compared to a control group without the multimedia presentations. Furthermore, the study showed that statistically, there was no difference in the exam scores for the control group and that of the students exposed the multimedia presentations.

From the exit interviews from students [2] there seemed to be two primary reasons for the dissatisfaction of the real-world examples in lecture. 1) The students did not see the examples as beneficial for their primary goal, which was surviving the

Digital Object Identifier (DOI):

http://dx.doi.org/10.18687/LACCEI2019.1.1.199

ISBN: 978-0-9993443-6-1 ISSN: 2414-6390 class itself. They would rather see more example problems to help them get a good grade in the class. 2) The students felt intimidated by the material presented. The examples included the bending of a fighter jet wing, a shaft of a jet engine in torsion, and the combined stresses on a human knee joint. Another study [3] showed the students were much less involved in class, actually putting down pencils and reclining in seats, during the multimedia portion of the lecture.

Instead of the instructor showing long multimedia examples and simulations from the front of the classroom, Beichner and Saul propose having the students work in groups with hands-on activities and simulations to teach themselves, with the instructor providing guidance and Socratic-type questioning [4]. Similar to a flipped classroom, the students are involved in activities to guide learning, while the majority of content comes from outside the classroom (reading, videos, etc). This studio-style classroom has shown to benefit all students in their ability to solve problems, overall attitudes, conceptual understanding and reducing failure rates, especially for underrepresented students.

Reinventing the Statics classroom [5] has some very useful manipulatives that could be used in the studio-style classroom to help students with fundamental concepts such as reaction forces and interconnecting bodies.

Applied Mechanics with SolidWorks is a book written because of an instructor observing students struggling to sketch the loci of a mechanism [6]. Onwubolu began creating a library of applied mechanics parts demonstrating how a mechanism moves and using SolidWorks to solve for motion problems. He used SolidWorks in several ways in the book to solve Statics and Dynamics problems. In addition to using SolidWorks Motion Analysis to solve the problems, he used the sketching tool to create velocity and force triangles, and to solve for vector addition and geometric analysis.

\section{SIMULATION SOLUTIONS}

Following are two tutorials using Creo Parametric ver. 4.0 to animate and solve problems found in the textbook, Vector Mechanics for Engineers, Dynamics, $11^{\text {th }}$ edition [7].

\section{A. Slide Block - Problem 12.35 [7]}

Block $B$ of mass $10-\mathrm{kg}$ rests as shown on the upper surface of a 22-kg wedge $A$ as shown in Fig. 1. Knowing that the system is released from rest and neglecting friction, determine (a) the acceleration of $B,(b)$ the velocity of $B$ relative to $A$ at $\mathrm{t}=0.5 \mathrm{~s}$. 


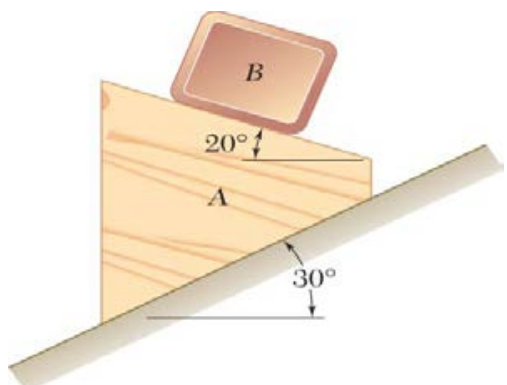

Fig. 1 Sliding Block B on Sliding Block A

1) Set up assembly with planar constraints between $A$ and incline (B and A). Assemble 12_35RAMP with Default constraint - making the ground immoveable (Fig. 2).

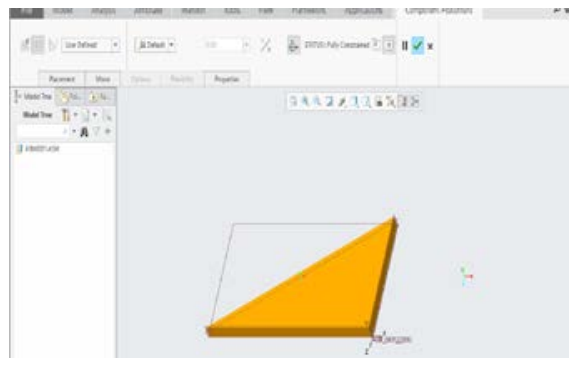

Fig. 2 Insertion of 12_35Ramp Part into Assembly

Next, assemble 12_35BLOCK_A with a Planar constraint selecting the inclined surface of ramp and surface of Block A with the axis on it (Fig. 3).

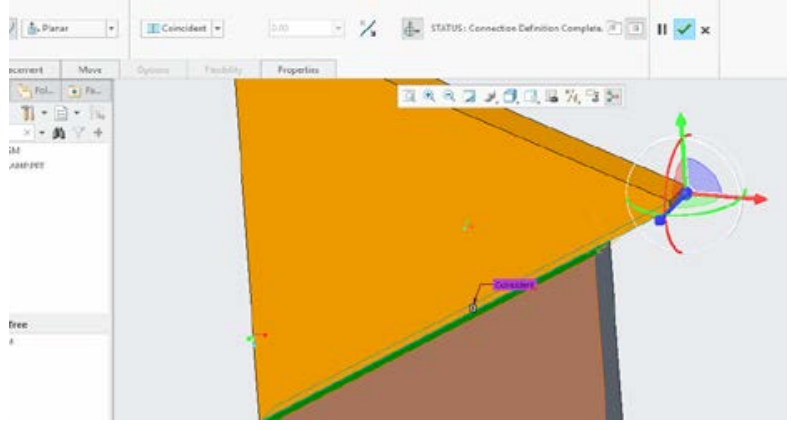

Fig. 3 Insertion of 12_35Block_A Part into Assembly

Similarly, assembly 12_35BLOCK_B with a planar constraint, selecting top surface now of Block A and surface with axis of Block B. Final assembly should look like Fig. 4. Notice that the proportions of the blocks and ramp are quite different than the problem given in the textbook [7].

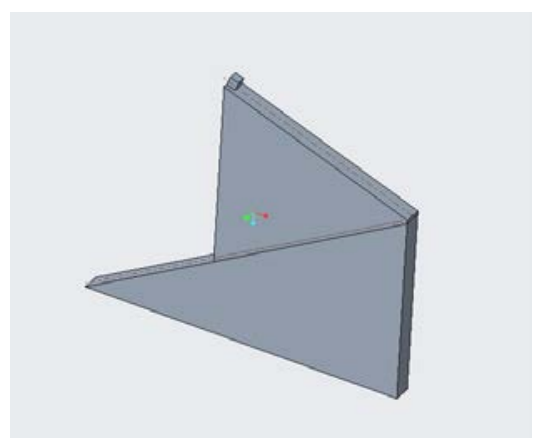

Fig. 4 Assembly of Sliding Block B on Sliding Block A

2) Create initial configuration shown in the problem (B at top of A).

Select Drag Components $>$ Snapshot $>$ Constraints and click on top box (Align two entities - see Fig. 5). Then select the axis on the ramp and the axis on connecting surface on Block A to align. Repeat for axes on top of Block A and connecting surface of block B. Continue to align so that Block A is aligned with top of ramp and Block B is aligned with top of Block A. After all is aligned, select Snapshot to "take a picture" of the configuration. This will be the starting configuration for the analysis (baseline).

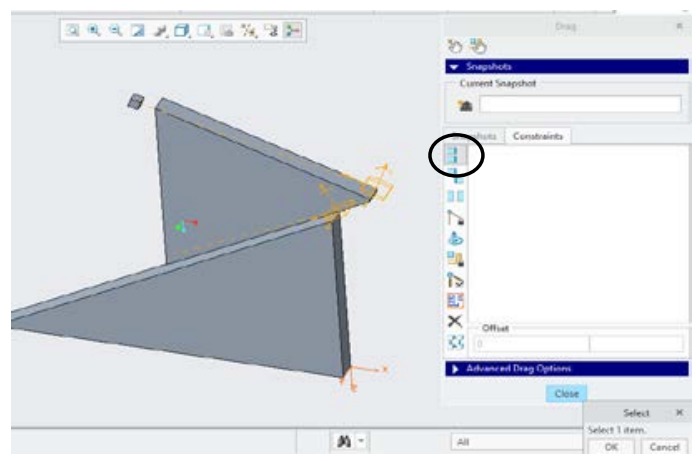

Fig. 5 Aligning blocks for initial configuration

3) Verify value of gravity - Enter Mechanism under Applications menu. Select Gravity (Properties and Conditions menu) and verify gravity is the magnitude and direction shown in the arrow below (Fig. 6). If the magnitude is in in $/ \sec ^{\wedge} \wedge 2-$ exit Mechanism, change units in Model Properties (File>Prepare>Model Properties) and return to Mechanism.

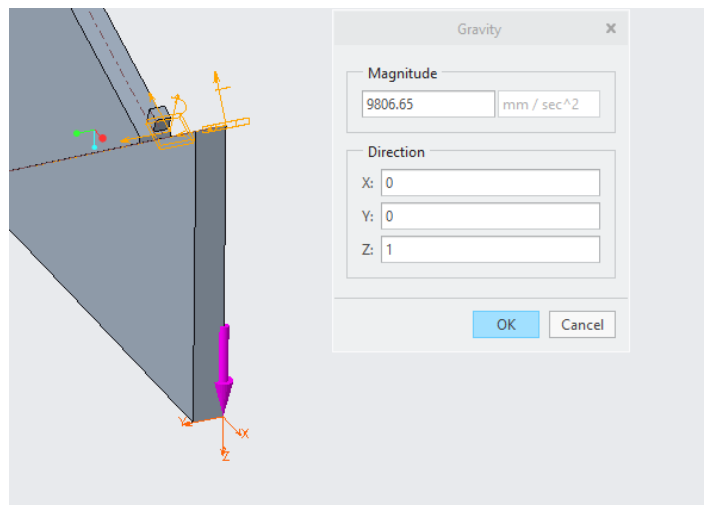

Fig. 6 Verifying Value for Gravity

17 ${ }^{\text {th }}$ LACCEI International Multi-Conference for Engineering, Education, and Technology: "Industry, Innovation, And Infrastructure for Sustainable Cities and Communities”, 24-26 July 2019, Jamaica. 
4) Dynamic analysis done with gravity enabled - Select Mechanism Analysis, under Type, select Dynamic (since this involves mass and gravity) and under External Loads tab, select box that reads "Enable Gravity” (Fig.7).

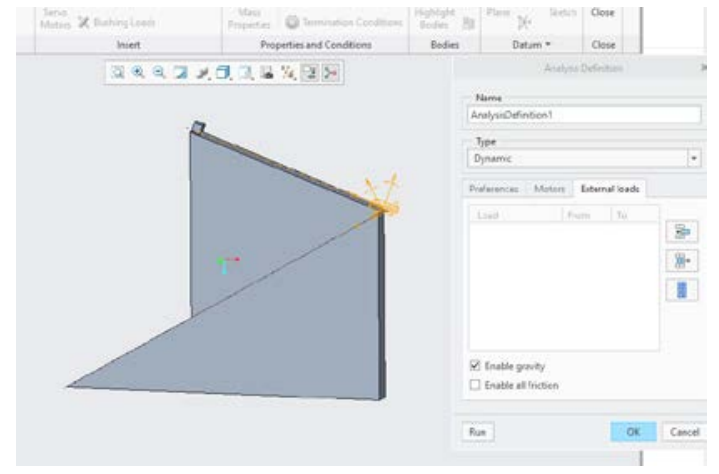

Fig. 7 Enable Gravity in Dynamic Mechanism Analysis

Under Preferences, input 2 seconds for Duration and 100 for Frame rate (see Fig. 8). Then select Run to run the analysis. Notice that even for only 2 seconds, the block completely slides off the screen.

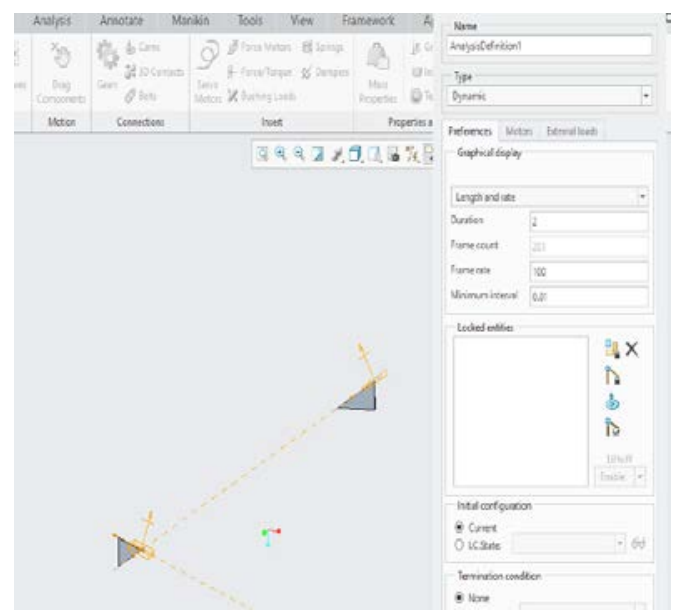

Fig. 8 Running the Analysis for Problem 12.35 [7]

5) Getting Measurements from Analysis

Measurements needed are 1) magnitude of acceleration of point on B using World Coordinate system and 2) magnitude of linear velocity of point on $B$ using coordinate system of part A (relative to A).

Under Analysis menu in Mechanism is Measures tool. Click on Measures tool to get the pop up menu shown in Fig. 9. Input vel_B for Name, select Velocity under Type, click on the select arrow for Point or Motion Axis and select PNTO point on Block B. For Coordinate System, click on select arrow and select coordinate system of Block A. This will give velocity of B with respect to A. If you wanted velocity of B with respect to ground, you would put World Coordinate System (of assembly) for the Coordinate System. Click on Apply and OK box to close.
Click on Measures again and this time input acc_B for Name, select Acceleration under Type, click on PNTO point on Block B for Point or Motion Axis and World Coordinate System. This will give acceleration of Block B with respect to ground.

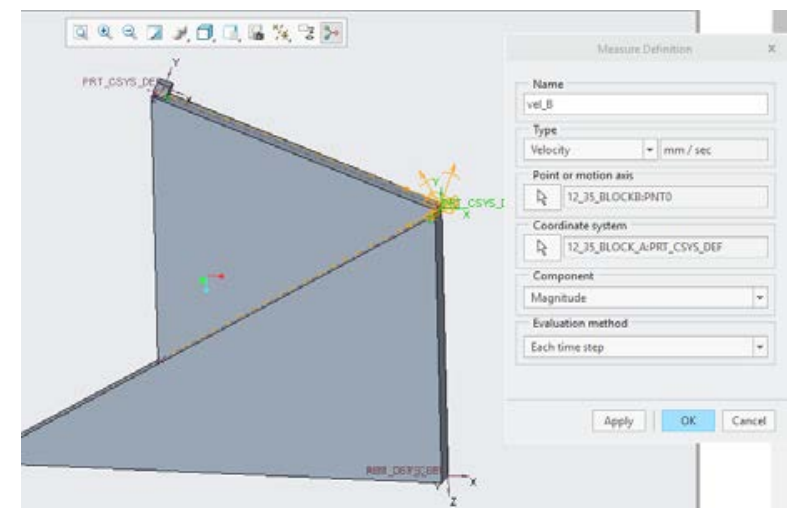

Fig. 9 Setting up to Measure Velocity of Block B

The Measures pop up menu should now look like Fig. 10. Select acc_B and AnalysisDefinition1 and click on the graph tool.

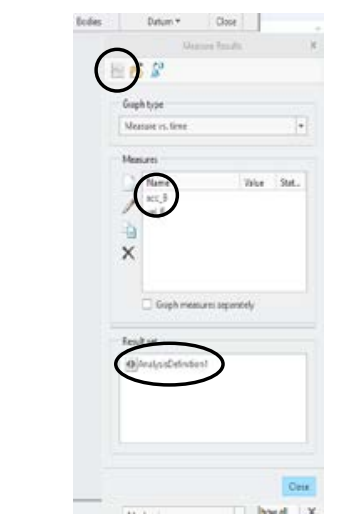

Fig. 10 Measures Pop-Up Menu

The graph for acceleration of B should look like Fig. 11 Title and Axis Label can be selected to change text. In selecting a data point, the calculated acceleration is 5942.69 $\mathrm{mm} / \mathrm{s}^{2}$ - which corresponds to the theoretical value (and answer in textbook [7]) of $5.94 \mathrm{~m} / \mathrm{s}^{2}$. 


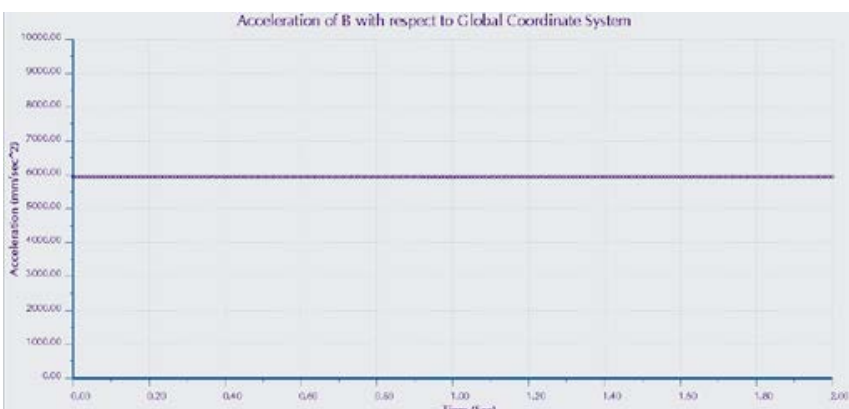

Fig. 11 Acceleration of B Graph from Mechanism Analysis

Similarly, the graph for velocity of block B is shown in Fig. 12.

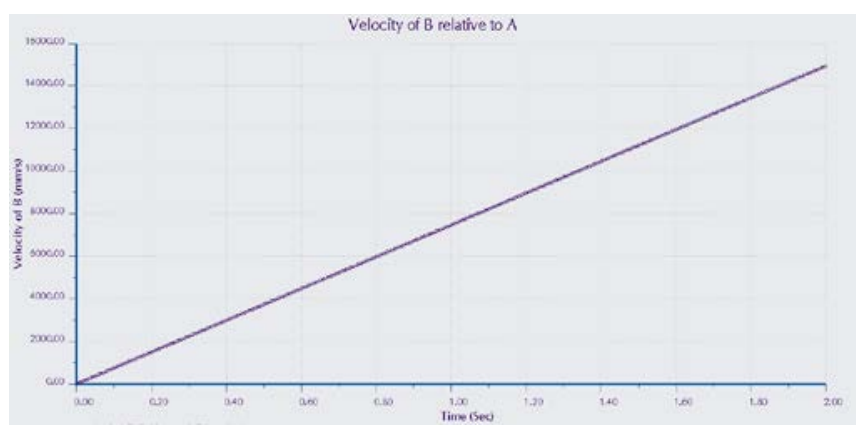

Fig. 12 Velocity of Block B Relative to A.

Data point on the graph corresponding to time 0.5 seconds, is $3735.22 \mathrm{~mm} / \mathrm{s}$. This corresponds to the theoretical (and textbook) answer of $3.74 \mathrm{~m} / \mathrm{s}$. Summary of theoretical and simulated results are given in Table 1.

Table 1 Summary of Results from Problem 12.35 [7]

\begin{tabular}{|l|l|l|l|}
\hline Variable & Theoretical & Simulation & \% Diff. \\
\hline $\mathrm{a}_{\mathrm{B}}$ & $5.94 \mathrm{~m} / \mathrm{s}$ & $5.94269 \mathrm{~m} / \mathrm{s}$ & $0.68 \%$ \\
\hline $\mathrm{V}_{\mathrm{B} / \mathrm{A}}$ & $3.74 \mathrm{~m} / \mathrm{s}$ & $3.73522 \mathrm{~m} / \mathrm{s}$ & $0.13 \%$ \\
\hline
\end{tabular}

\section{B. $\quad$ Oscillating Spring - Problem 13.93 [7]}

Collar $A$ has a mass of $3 \mathrm{~kg}$ and is attached to a spring of constant $1200 \mathrm{~N} / \mathrm{m}$ and of undeformed length equal to $0.5 \mathrm{~m}$ as shown in Fig. 13. The system is set in motion with $r=0.3 \mathrm{~m}$, $v_{\theta}=2 \mathrm{~m} / \mathrm{s}$, and $v_{r}=0$. Neglecting the mass of the rod and the effect of friction, determine the radial and transverse components of the velocity of the collar when $r=0.6 \mathrm{~m}$.

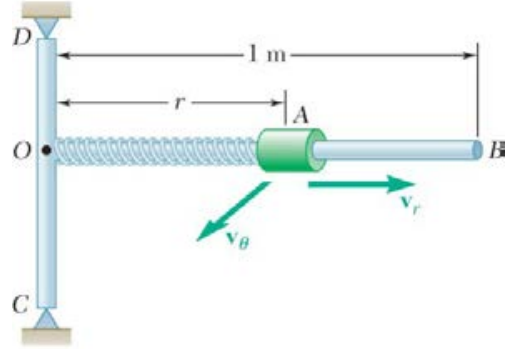

Fig. 13 Oscillating Spring Problem 13.93 [7]

1) Create axis and point in assembly for pin joint. Next to Model Tree in a new assembly, select Settings $>$ Tree Filters (Fig. 14) and click on Features in Display so box is checked. This will allow you to see planes, axes and points at the assembly level instead of just parts in the Model Tree.

Under Model Table and Datum Menu, select Axis to create an axis. In the pop-up menu (Fig. 15) select assembly front plane as a reference and while holding down control key on keyboard, input right plane in same reference box.

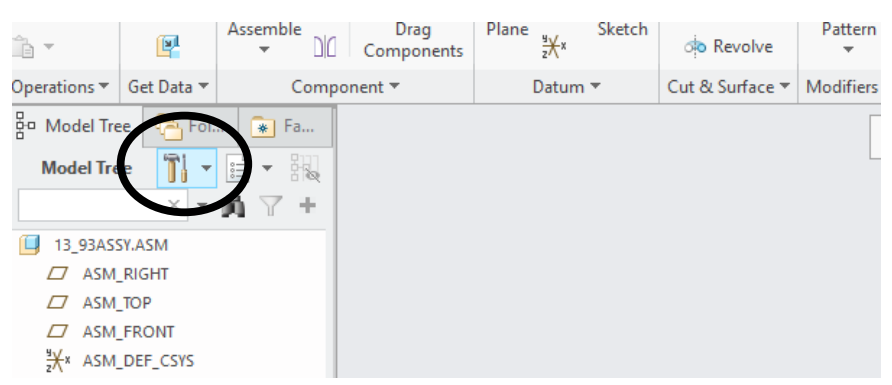

Fig. 14 Settings in Model Tree

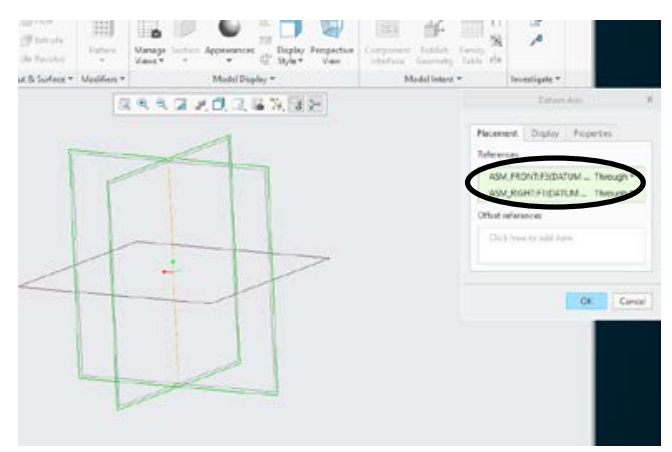

Fig. 15 Creating an Axis in the Assembly

Select Create Point in Datum Menu of Model tab and input AA_1 (axis just created) and Top Plane for references (control key pressed simultaneously) 
2) Set up assembly with pin and slider joints. Select Assemble under Component window of Model tab and find 13_99_FRAME part. Select Pin Joint Connection and select axis through vertical portion (CD in textbook) and assembly axis AA_1 (see Fig. 16). For Translation constraint, select PNTO from the frame and APNTO from the assembly.

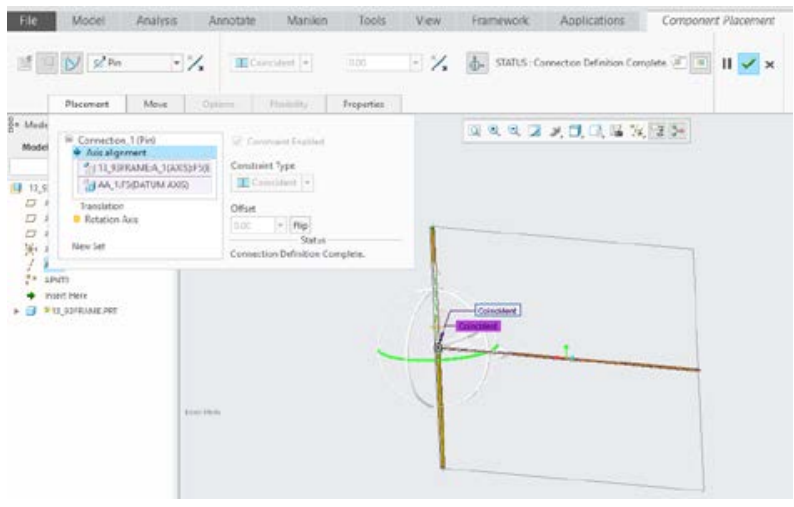

Fig. 16 Inserting Frame into Assembly

Assemble 13_93COLLAR with a Slider Connection, aligning A_1 on collar to A_2 (horizontal axis on FRAME). Rotational constraint should be Front Plane of COLLAR and Top Plane of FRAME. There is no translation constraint.

3) Create spring attached at $\mathrm{O}$ and on collar, $\mathrm{k}=1200 \mathrm{~N} / \mathrm{m}$ and undeformed length $=0.5 \mathrm{~m}$. To make a spring, select springs from Insert Menu of Mechanism tab (Fig. 17).

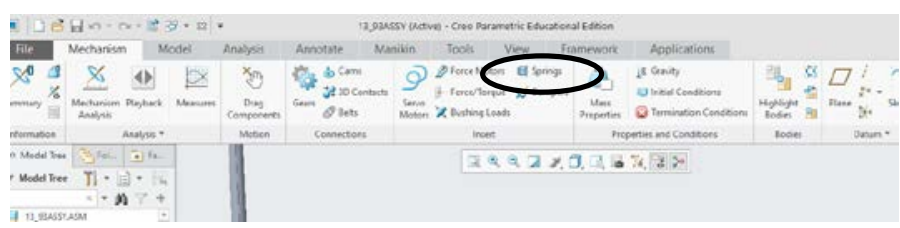

Fig. 17 Inserting a Spring into the Assembly

Click on PNTO of COLLAR and APNTO of Assembly, then input $1200 \mathrm{~N} / \mathrm{m}$ for $\mathrm{K}$ and $500 \mathrm{~mm}$ for $\mathrm{U}$ (undeformed length) (Fig. 18). To change diameter of spring, click options (does not affect simulation).

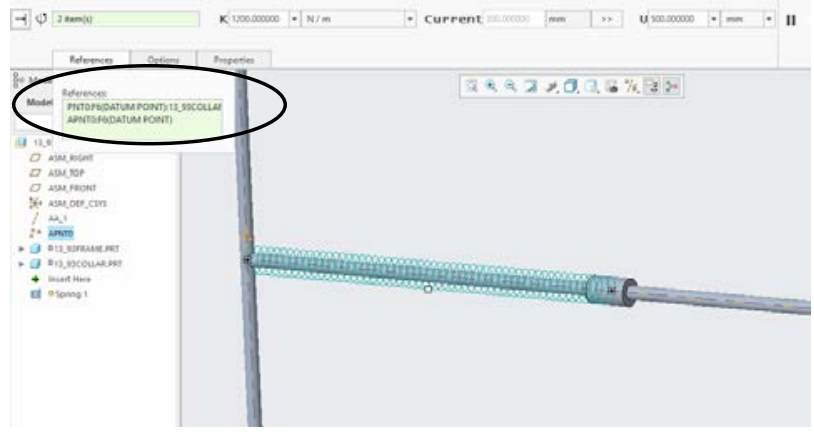

Fig. 18 Configuring the Spring in the Assembly
4) Create initial configuration with collar $0.3 \mathrm{~m}$ from rotation axis (CD). Under Mechanism tab, select Drag Components. Click on Snapshot, then Constraints and Align Entities. Click on PNTO of COLLAR and PTN1 of FRAME ( $0.3 \mathrm{~m}$ from rotation axis) to align (Fig. 19). Once aligned, click on camera to take a Snapshot of configuration.

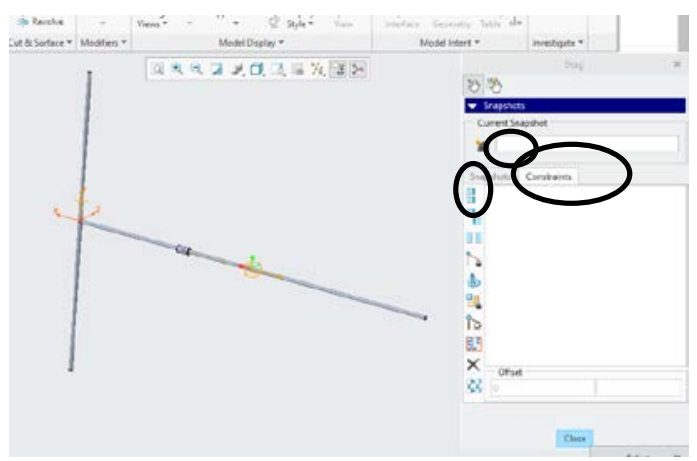

Fig. 19 Setting Initial Position

5) Create initial conditions of $v_{1 \theta}=2 \mathrm{~m} / \mathrm{s}$ and $v_{1 \mathrm{r}}=0$. Under Properties and Conditions menu of Mechanism tab, click on Initial Conditions (Fig. 20). Click on Define velocity of a point - and select PTNO on COLLAR

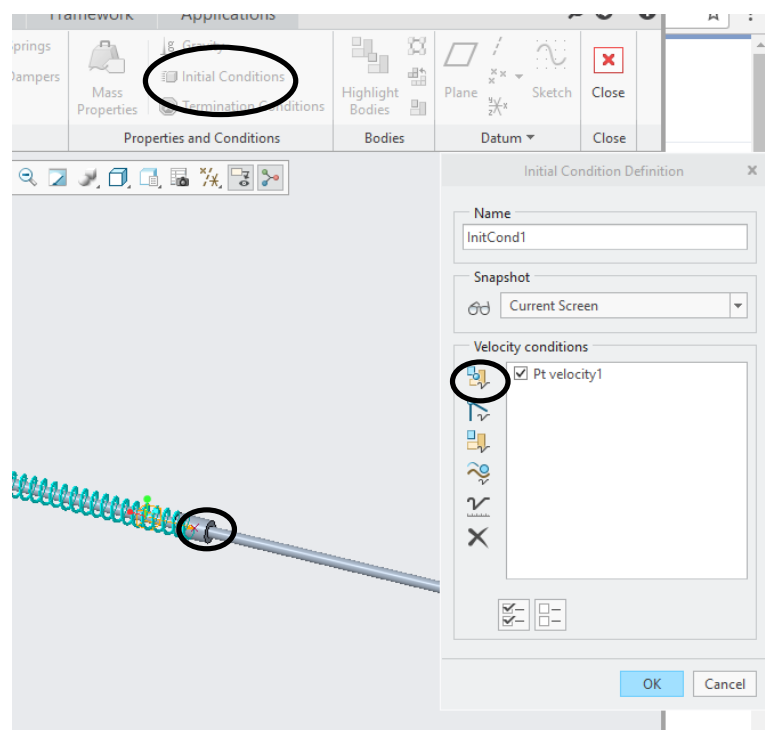

Fig. 20 Inputting Initial Conditions. 
Input a magnitude of $2000 \mathrm{~mm} / \mathrm{sec}$ and select the coordinate direction so arrow is in correct direction as shown in Fig.21.

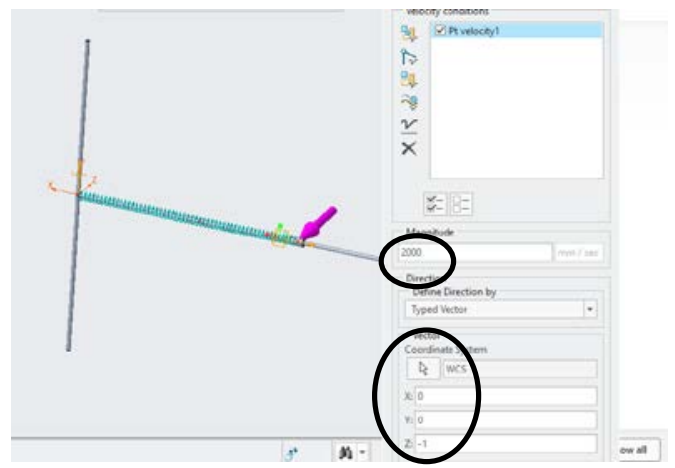

Fig. 21 Inputting Initial Conditions - Velocity

The other initial condition is $\mathrm{v}_{\mathrm{r}}=0$, so no input is needed. (If it was a value, another velocity of the point on the collar would be defined then input the desired magnitude.)

6) Dynamic analysis with initial conditions. Select Mechanism Analysis from Analysis menu of Mechanism tab. As shown in Fig. 22, for Type of analysis = Dynamic, for 1 second duration and 1000 frames/s. Make sure IC State in Initial Configuration is set to the initial conditions created previously. Run the analysis.

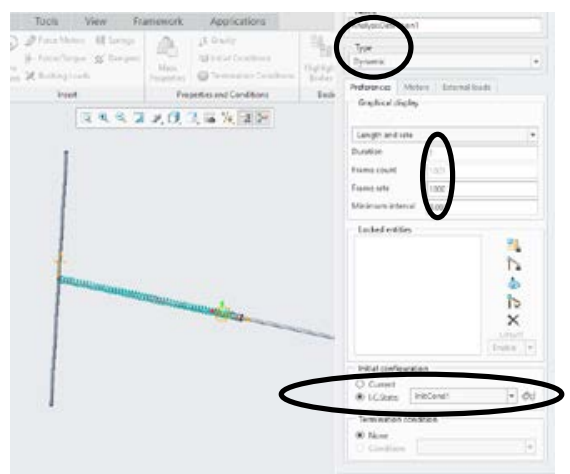

Fig. 22 Running the Dynamic Analysis

7) Measures for this problem are the position of collar from axis of rotation, the radial velocity and the angular velocity. Set up for radial position is found in Fig. 23. The time for when radial position reaches $0.6 \mathrm{~m}$ will be observed on graph in Fig. 24.

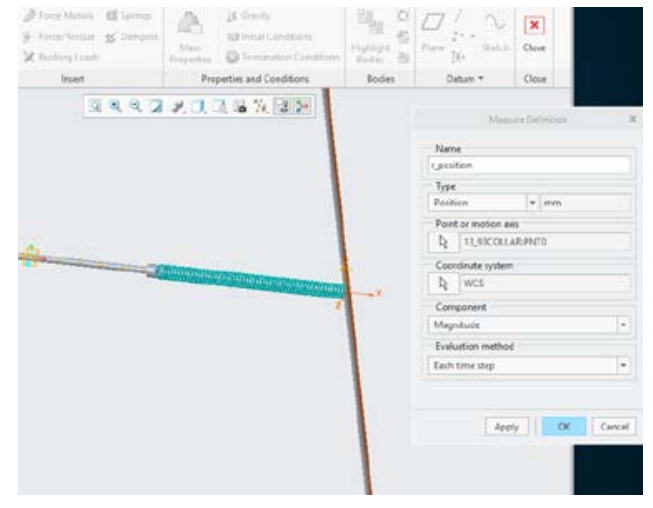

Fig. 23 Defining the Radial Position Measure

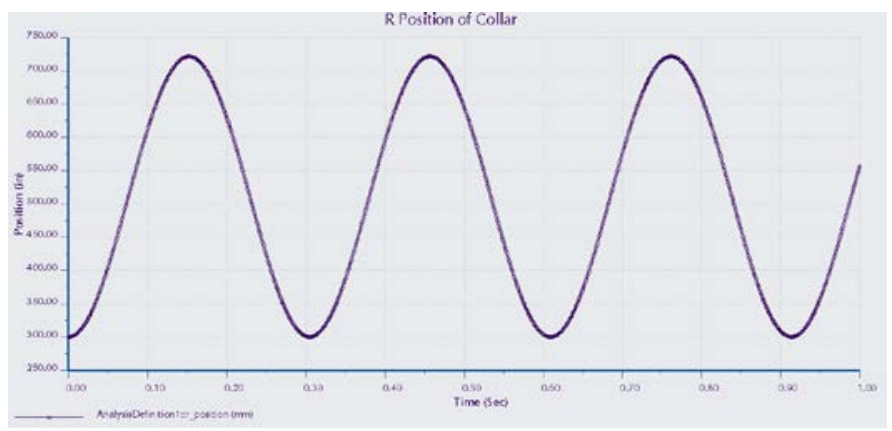

Fig. 24 Graph of Radial Position of Collar

Reading from the graph (Fig. 24), the radial position of 600.972 mm occurs at time $=0.097 \mathrm{~s}$.

Fig. 25 shows the set up of the radial velocity measure, notice that the coordinate axis is CS0 from the frame. The graph of radial velocity (Fig. 26) then shows at time $=0.097 \mathrm{~s}$ (when $\mathrm{r} \sim$ $0.6 \mathrm{~m}$ ) the radial velocity is $3896.4 \mathrm{~mm} / \mathrm{s}$.

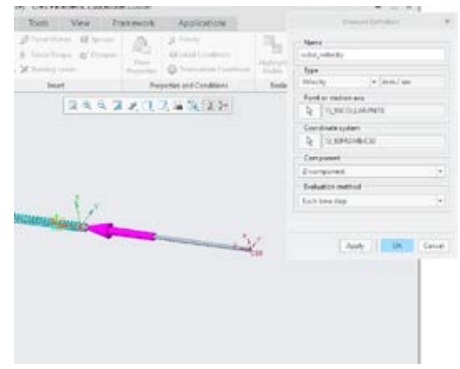

Fig. 25 Defining the Radial Velocity Measure

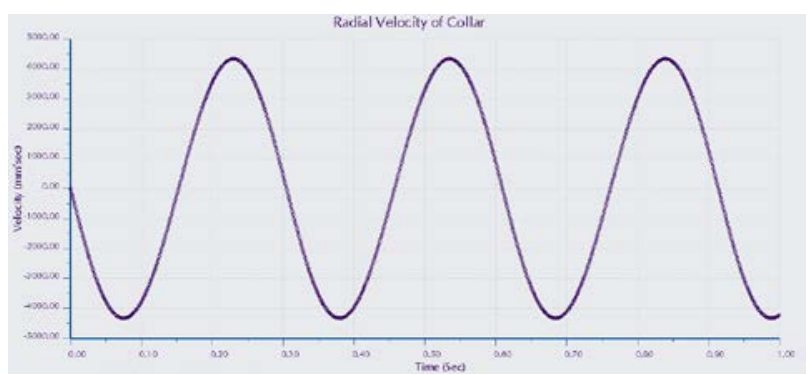

Fig. 26 Graph of Radial Velocity

$1^{\text {th }}$ LACCEI International Multi-Conference for Engineering, Education, and Technology: "Industry, Innovation, And Infrastructure for Sustainable Cities and Communities”, 24-26 July 2019, Jamaica. 
From the theoretical solution, $\mathrm{v}_{\mathrm{R}}=+/-3.87 \mathrm{~m} / \mathrm{s}$. The value from the simulation is $0.68 \%$ off from theoretical - possibly because at $\mathrm{t}=0.097$, the $\mathrm{r}$ value was not exactly $600 \mathrm{~mm}$ (Table 2 ).

The other measure for this analysis is the velocity in the theta direction. This is accomplished by measuring the angular velocity of the pin joint connection (Fig. 27) and multiplying the acquired number by the $r$ position value at the time of interest ( $600 \mathrm{~mm})$. This is from equation:

$$
\begin{gathered}
\mathrm{v}=\mathrm{r} \omega \\
\text { At } \mathrm{t}=0.097 \mathrm{~s}, \omega=103.336 \mathrm{deg} / \mathrm{s}(1.80355 \mathrm{rad} / \mathrm{s}) \\
\text { (from Fig. } 28) \\
\mathrm{V}_{\theta}=600.972 \mathrm{~mm}(1.80355 \mathrm{rad} / \mathrm{s})=1083.88 \mathrm{~mm} / \mathrm{s}
\end{gathered}
$$

From theoretical solution, $\mathrm{v}_{\theta}=1 \mathrm{~m} / \mathrm{s}$, so simulated value is $8.4 \%$ from theoretical value.

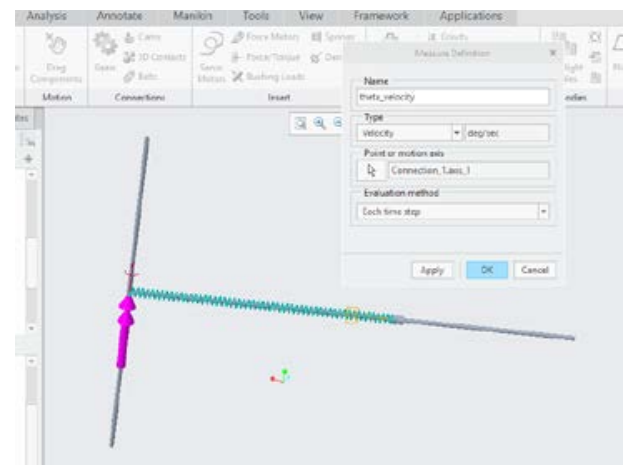

Fig. 27 Defining the Angular Velocity Measurement

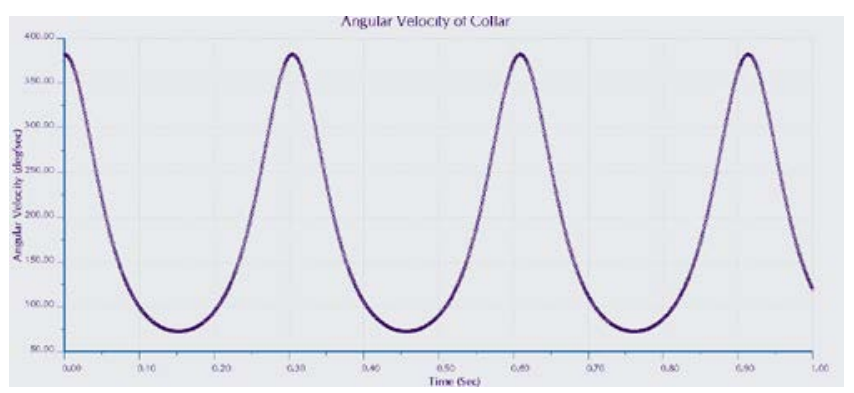

Fig. 28 Graph of Angular Velocity

Table 2 Summary of Results from Problem 13.93 [7]

\begin{tabular}{|l|l|l|l|}
\hline Variable & Theoretical & Simulation & \%Diff. \\
\hline $\mathrm{v}_{\mathrm{r}}$ & $3.87 \mathrm{~m} / \mathrm{s}$ & $3.8964 \mathrm{~m} / \mathrm{s}$ & $0.68 \%$ \\
\hline $\mathrm{v}_{\theta}$ & $1 \mathrm{~m} / \mathrm{s}$ & $1.08388 \mathrm{~m} / \mathrm{s}$ & $8.4 \%$ \\
\hline
\end{tabular}

\section{OBSERVATION AND CHALLENGES}

Problem 12.35 was chosen to help the student visualize the relative motion between blocks $\mathrm{A}$ and $\mathrm{B}$. The unexpected challenge of this problem was that no other dimensions than the angles were given. If the parts are created to look like the problem shown in the textbook, with gravity and no friction, block B quickly slides off block A and "hits" the incline (actually falls through it in simulation). The actual scale of block A and B are much larger than it appears in the book as shown in tutorial.

Problem 13.93 was chosen to show the oscillation that occurs from the spring as the rod CD is rotating. The challenge in the simulation is finding the correct coordinate axis for the measures of velocity. Since $A$ is rotating about point $O$, the radial velocity must be measured with respect to a point on the rotating bar (point B for example).

\section{CONCLUSION}

Statics and Dynamics are essential fundamental courses for engineering. The prerequisites of Calculus and Physics are necessary for manipulating and solving vector problems, but often the students lack the 3D spatial visualization skills needed to understand the engineering problems. Although the mathematics and physics may be challenging for some students, the real obstacle arise in the visualization of the problem. Most students struggle to determine, for example, what is happening in the problem, reading dimensions, and finding needed angles for calculations. This instructional tutorial was designed to provide much needed insight on utilizing parametric modeling to aid in visualization of engineering problems. The hope is that these techniques and practices can be expanded to help better understand engineering problems and improve success rates of students, especially underrepresented students.

\section{REFERENCES}

[1] Milne, M. \& Katz, T. (2017) Assessing The 3D Visualisation Skills Of Engineering Students And Developing Techniques For Support. Journal of Education. 345(6) pp/554-567.

[2] Bowe, M., Jensen, D., Feland, J., Self, B. (2001) "When Multimedia Doesn't Work: An Assessment of Visualization Modules for Learning Enhancement in Mechanics", Proceedings of the ASEE Annual Conference, Albuquerque, NM.

[3] Hubing, $\mathrm{N}$ et al (2002) Interactive Learning Tools: Animating Statics, American Society for Engineering Education Annual Conference \& Exposition.

[4] Beichner, R. \& Saul, J. (2003) Introduction to the SCALE-UP (StudentCentered Activities for Large Enrollment Undergraduate Programs) Project, Proceedings of the International School of Physics, Varenna, Italy.

[5] P. F. Steif \& A. Dollar (2005), Reinventing the Teaching of Statics, Int. J. Engng. Ed. 21(4), pp. $723-729$.

[6] Onwubolu, G. C. (2015) Applied Mechanics with Solidworks.

[7] Beer, F. P., Johnston, E. R., Eisenberg, E. R., \& Sarubbi, R. G. (1997). Vector mechanics for engineers. New York: McGraw-Hill.

$1^{\text {th }}$ LACCEI International Multi-Conference for Engineering, Education, and Technology: "Industry, Innovation, And Infrastructure for Sustainable Cities and Communities”, 24-26 July 2019, Jamaica. 